\title{
Utilidad neta y generación de valor económico agregado, en empresas del sector minero del Perú, periodo 2012-2016
}

\author{
Net income and generation of added economic value, in companies in the mining \\ sector of Peru, during the 2012-2016 time period
}

\author{
Samuel Yair Paredes Cárdenas ${ }^{1}$ \\ ${ }^{1}$ Escuela Profesional Contabilidad, Facultad de Ciencias Empresariales, Universidad Peruana Unión
}

INFORMACIÓN DEL ARTÍCULO

Historia del artículo

Recibido: 14 de octubre 2017

Aceptado: 28 de enero 2018
Palabras clave:

relación, utilidad neta, valor económico agregado (EVA).

\section{Resumen}

La presente investigación determina el grado de relación que existe entre la utilidad neta y la generación de valor económico agregado (EVA), en el sector minero. La metodología utilizada dentro del enfoque cuantitativo, fue de tipo correlacional bajo un diseño no experimental; para el alcance de los resultados se determinó una muestra de estudio por conveniencia de 16 estados de resultados de empresas del sector minero que cotizaron permanentemente en la bolsa de valores del Perú durante el periodo comprendido desde el año 2012 al 2016. Asimismo, se tuvieron en consideración los valores que independientemente cada empresa obtuvo en cada periodo tanto para la utilidad neta como para el EVA y a partir de ello se agruparon de manera sectorial. Los resultados determinaron que existe un grado de relación estadísticamente significativa positiva muy alta entre la utilidad neta y el EVA para cada uno de los periodos correspondientes $(r=.988)$. Por tanto, a lo largo del periodo comprendido desde el 2012 al 2016 en las empresas del sector minero que cotizan en la Bolsa de Valores de Lima, la utilidad neta obtenida por las empresas estuvo directamente relacionada a la generación de valor económico.

\section{Abstract}

This research study determines the degree of relationship between net income and the generation of economic value added (EVA) in the mining sector. The methodology used within the quantitative approach was correlational with a non-experimental design. For the scope of the results, a study sample was determined by convenience including 16 income statements of companies in the mining sector that were permanently traded on the stock exchange of Peru during the period 2012 to 2016. Likewise, the values that were independently taken into account for each company for each period were both net income and EVA and from this they were grouped in a sectorial way. The results show that there is a very high degree of significant positive relationship between net income and the EVA for each of the corresponding periods $(r=.988)$. Therefore, throughout the period 2012 to 2016 in the companies of the mining sector that were listed on the Lima Stock Exchange, the net profit obtained by the companies was directly related to the generation of economic value. relationship, net profit, economic value added (EVA). 


\section{Introducción}

La minería es el eje principal de la economía peruana, es así que, como país Perú ha logrado un sitial importante en la producción minera mundial, ubicándose entre los primeros países productores de plata, cobre, zinc, estaño, plomo y oro (Benavidez, 2012). De allí que, el crecimiento en la producción de metales se ha visto reflejada en la utilidad neta como un beneficio económico periódico creciente en los últimos años, siendo de vital importancia porque marca el ritmo del progreso o desarrollo empresarial.

A pesar de que los resultados financieros son de gran utilidad para situaciones trascendentales, como apalancamiento financiero, emisión de nuevas acciones, pago del impuesto a la renta, entre otras, el beneficio económico como tal no muestra en sus resultados la generación de valor económico agregado para dicho sector.

Por su parte, el resultado financiero EVA sí permite determinar la generación de valor económico agregado para las empresas a partir del beneficio económico generado por las mismas (Li Bonilla, 2018); sin embargo, requiere de puntos específicos de información que muchas veces no están específicamente detallados en los estados financieros presentados a la SMV; cosa que no limita la determinación adecuada del EVA, pero disminuye un porcentaje de su resultado óptimo.

Ahora bien, siendo que el EVA es una herramienta alternativa de evaluación financiera utilizada por grandes empresas y corporaciones la cual guarda una importante relación con el beneficio económico de una empresa a partir de su utilidad neta, y el costo de financiamiento de la inversión total incluyendo el financiamiento de terceros; surge la inquietud de determinar si existe relación entre la utilidad neta y la generación de valor económico agregado, en las empresas del sector minero que cotizan en bolsa de valores del Perú durante el periodo 2012 al 2016.

\section{Referencias teóricas}

\section{Los estados financieros}

Según Ortiz (2006), los estados financieros constituyen una representación estructurada de la situación financiera y del rendimiento financiero de una entidad; de allí que tienen el propósito de suministrar información acerca del ambiente financiero, así como del rendimiento financiero y de los flujos de efectivo de la entidad; permitiendo su utilidad a la hora de tomar decisiones económicas, debido a que muestran los resultados de la gestión realizada por los administradores con los recursos que se les ha confiado.

\section{El estado de situación financiera}

El estado de situación financiera es una representación de dualidad económica en la entidad. Ahora bien, independientemente de que, al preparar un balance general, se debe obtener igualdad entre los recursos y sus fuentes; la finalidad del estado no solo es igualar las partidas, sino que pretende mostrar la posición o situación de la entidad en una fecha determinada.

Además, de su estudio, análisis e interpretación; los responsables de tomar decisiones pueden conocer la liquidez 0 capacidad de pago inmediato o a corto plazo; es decir, determinar la posibilidad que la empresa tiene para hacer frente a 
sus compromisos u obligaciones financieros a corto plazo.

\section{El estado de resultados integrales}

Estupiñan (2006), afirma que el estado de resultados, muestra la información relativa al resultado de sus operaciones en un periodo y, por ende, de los ingresos, gastos; así como, de la utilidad (pérdida), neta o cambio neto en el patrimonio contable resultante en el periodo.

Por su parte, Urias (1995), afirma que el reporte del estado de resultados, permite conocer el balance general dentro del capital ganado, contra la inversión o recursos que muestra el estado; determinando si la empresa es rentable o no para los interesados en invertir; es decir, muestra si se trata de un buen o mal negocio; ya que evidencia el porcentaje de los recursos del negocio que ha sido aportado por los acreedores y por los propietarios, para conocer quién posee realmente la entidad y prevenir los riesgos de que la empresa quede en manos de los acreedores; mostrando así el rendimiento o rentabilidad sobre la inversión.

\section{Utilidad neta}

La utilidad neta es el valor residual de los ingresos de una entidad lucrativa, después de haber disminuido sus costos y gastos relativos, reconocidos en el estado de resultados siempre que estos últimos sean menores a dichos ingresos, durante un periodo contable. Caso contrario, la resultante es una pérdida neta. Por tanto, la empresa obtiene una utilidad neta si los ingresos son mayores que sus gastos, pero si los gastos son mayores, tendrá una pérdida neta (Morales, 2011).

Valor económico agregado (EVA)
El EVA es una variación de lo que tradicionalmente se ha llamado ingreso o beneficio residual. Según Dodd (1996), es el resultado que se obtenía al restar a la utilidad operacional, los costos de capital; resaltando la idea de que el beneficio residual apareció en la literatura de teoría contable de las primeras décadas de este siglo; es así que inicialmente, lo introdujo Church en 1917 y, posteriormente, Scovell en 1924, más adelante emerge en la literatura de contabilidad gerencial en los años sesenta; pero no es sino hasta 1975 que Virtanen lo define como un complemento del retorno sobre la inversión para la toma de decisiones, no obstante, establece que algunos estudiosos finlandeses ya habían discutido dicho concepto a principios de los setenta. Bajo este criterio el EVA se calcula de la siguiente manera:

$$
E V A=B A I D I-V C A \times C P P C \circ \text { WACC }
$$

\section{Donde:}

EVA: Valor económico agregado.

BAIDI: Beneficio antes de intereses y después de impuestos.

VCA: Valor Contable del Activo.

CPPC: Costo Promedio Ponderado de Capital.

\section{EI UAIDI O BAIDI}

Es una de las tres grandes variables que inciden en el EVA que genera una empresa y, sin duda una de las formas de aumentar este valor es incrementando este resultado. Los beneficios antes de intereses y después de impuestos (BAIDI), excluyen las utilidades extraordinarias para concentrarse en las que son consecuencia de la actividad típica de la empresa. Se puede obtener a partir de la utilidad neta incorporando los gastos financieros y restando las utilidades extraordinarias. (Backes, 2002). 


\section{El valor contable del activo (VCA)}

Para el cálculo del activo para el EVA Amat O. (1999), recomienda:

Activos a valor de adquisición

(-) Amortizaciones del activo fijo

(+) Plusvalías producidas en los activos

(-) Minusvalías producidas en los activos

(-) Financiación automática de proveedores, hacienda pública o impuestos, seguridad social.

(=) Activo neto (valor de mercado)

Amat (1999), indica que, el valor del activo corresponde al valor de adquisición, una vez deducidas las depreciaciones correspondientes, de allí que los activos a considerar, pueden ser los existentes a principio del ejercicio, los existentes al final, o un promedio. Por tanto, el valor más extendido entre las empresas que usan el EVA es del promedio.

El costo promedio ponderado del capital (CPPC) o el WACC

Navarro (2017), manifiesta que el costo promedio de capital en finanzas, es uno de los indicadores más importantes el cual permite conocer cuánto le cuesta a la empresa financiarse con terceros, con patrimonio o utilizando ambas opciones; por ello debe existir una mezcla de financiamiento. Esto permitiría a la empresa financiarse totalmente con patrimonio, como también una parte con deuda y una parte con patrimonio; precisamente permite calcular ese costo promedio de capital que es la mezcla de financiación entre terceros y dueños.

$$
K e\left(\frac{P}{D+P}\right)+K d(1-T I) *\left(\frac{D}{D+P}\right)
$$

Donde:

$\mathrm{Ke}=$ Tasa del costo del patrimonio

$\mathrm{P}=$ Patrimonio

$\mathrm{D}=$ Deuda

$\mathrm{Kd}=$ Tasa del costo de la deuda

$\mathrm{TI}=$ Tasa de impuestos a la renta .

Al tasar el coste del patrimonio (ke), se utiliza usualmente el Capital Asset Pricing Model, o CAPM. El cual sirve para calcular el precio de un activo o una cartera de inversión y supone la existencia de una relación lineal entre el riesgo y la rentabilidad financiera del activo, donde:

$K e=T L R f+[B[T P M f-T L R f]+R \cdot P$

\section{Donde:}

Ke: Coste del patrimonio

TLRf: Tasa libre de riesgo

B: Riesgo de mercado de un activo

El costo de capital que se incluye en el cálculo del EVA equivale al concepto de costo de oportunidad (Copeland, 1990).

\section{Metodología}

La investigación es de tipo cuantitativa con un alcance correlacional, puesto que se trabajan con valores numéricos y busca determinar la relación que existe entre las variables de estudio (Hernandez y Baptista, 2010). Para el análisis de los resultados se consideró un total de 16 empresas pertenecientes al sector minero, de los periodos 2012 al 2016, publicados en el portal de la Bolsa de valo- 
res de Lima; asimismo, se utilizó el estadístico de prueba paramétrica coeficiente de correlación de Pearson.

\section{Resultados}

\section{Análisis descriptivos}

Considerando la Tabla 1 de resultados acumulados obtenidos por las empresas pertenecientes al sector minero desde el periodo 2012 al 2016, y adicionando los cuadros de reporte de estimación de diferencias absolutas y relativas; se evidencia que las empresas dentro de los resultados acumulados de los 5 periodos al comparar la utilidad neta, el EVA tuvo una diferencia absoluta de $\$ 6.250$ '259 miles de dólarespor debajo de la utilidad neta con una diferencia relativa del $87.94 \%$ menor que la utilidad neta; lo que implica que el sector minero en sus resultados acumulados a pesar de tener un beneficio económico a través de la utilidad neta no generó valor económico agregado al mismo nivel que el beneficio económico obtenido; por tal motivo, el sector minero desde el periodo 2012 al 2016 generó un valor económico agregado equivalente al $12.06 \%$ de su beneficio económico acumulado (utilidad neta acumulada).

Tabla 1

Resultados acumulados 2012 al 2016

\begin{tabular}{lccccccc}
\hline PERIODO & UTILIDAD & BAIDI & VCA & WACC & EVA & Dif.Absoluta & Dif.Relativa \\
\hline Sector M. 2012 & $2^{\prime} 916,911$ & $2^{\prime} 990,307$ & $15^{\prime} 811,488$ & $7.26 \%$ & $1^{\prime} 798,866$ & $1^{\prime} 118,045$ & $38.33 \%$ \\
Sector M. 2013 & $2^{\prime} 139,056$ & $2^{\prime} 264,249$ & $17^{\prime} 586,097$ & $7.87 \%$ & 832,048 & $1^{\prime} 307,008$ & $61.10 \%$ \\
Sector M. 2014 & $1^{\prime} 314,392$ & $1^{\prime} 507,643$ & $19^{\prime} 542,358$ & $8.61 \%$ & $-225,668$ & $1^{\prime} 540,060$ & $117.17 \%$ \\
Sector M.2015 & 41,446 & 368,791 & $20^{\prime} 439,907$ & $7.28 \%$ & $-1^{\prime} 085,194$ & $1^{\prime} 126,640$ & $2718.33 \%$ \\
Sector M.2016 & 695,233 & $1^{\prime} 010,382$ & $20^{\prime} 506,470$ & $7.38 \%$ & $-463,273$ & $1^{\prime} 158,506$ & $166.64 \%$ \\
\hline 2012 -2016 & $7^{\prime} 107,038$ & $8^{\prime} 141,372$ & $93^{\prime} 886,320$ & $7.68 \%$ & 856,779 & $6^{\prime} 250,259$ & $87.94 \%$ \\
\hline
\end{tabular}

De acuerdo con los resultados obtenidos en la Tabla 2, en comparación con la utilidad neta; el EVA tuvo una diferencia absoluta de $\$ 1,118$ '045 miles de dólares por debajo de la utilidad neta y una diferencia relativa del $38.33 \%$ menor que la utilidad neta; el sector minero en sus resultados acumulados a pesar de tener be- neficio económico a través de la utilidad neta no generó valor económico agregado, al mismo nivel que el beneficio económico obtenido; por tal motivo, el sector minero en el periodo 2012 genero un valor económico agregado equivalente al $61.67 \%$ de su beneficio económico acumulado (utilidad neta acumulada). 
Tabla 2

Promedio Resultados con variación relativa y absoluta 2012

\begin{tabular}{|c|c|c|c|c|c|c|c|}
\hline Empresas & Utilidad & BAIDI & VCA & WACC & EVA & $\begin{array}{c}\text { Dif. } \\
\text { Absoluta }\end{array}$ & $\begin{array}{c}\text { Dif. } \\
\text { Relativa }\end{array}$ \\
\hline $\begin{array}{l}\text { Southern Peru Cooper } \\
\text { Corporation }\end{array}$ & 990,049 & 995,579 & $2,865,733$ & $8.10 \%$ & 763,563 & 226,486 & $22.88 \%$ \\
\hline Shoughan Hierro Peru S.A. & 241,141 & 245,640 & 675,785 & $5.52 \%$ & 208,336 & 32,805 & $13.60 \%$ \\
\hline $\begin{array}{l}\text { Volcan Compañía Minera } \\
\text { S.A.A. }\end{array}$ & 237,548 & 279,924 & $2,133,648$ & $6.37 \%$ & 144,117 & 93,431 & $39.33 \%$ \\
\hline $\begin{array}{l}\text { Sociedad Minera Cerro Verde } \\
\text { S.A. }\end{array}$ & 772,070 & 775,872 & $3,449,708$ & $8.12 \%$ & 495,756 & 276,314 & $35.79 \%$ \\
\hline Compañia Minera Milpo S.A. & 20,058 & 25,692 & 665,090 & $6.13 \%$ & $-15,106$ & 35,164 & $175.31 \%$ \\
\hline $\begin{array}{l}\text { Compañia Minera Poderosa } \\
\text { S.A. }\end{array}$ & 44,070 & 43,997 & 167,429 & $7.32 \%$ & 31,742 & 12,328 & $27.97 \%$ \\
\hline Sociedad Minera Corona S.A. & 47,711 & 49,062 & 100,595 & $7.24 \%$ & 41,778 & 5,933 & $12.44 \%$ \\
\hline $\begin{array}{l}\text { Minera Andina de } \\
\text { Exploraciones S.A.A. }\end{array}$ & -51 & 60 & 1,182 & $8.12 \%$ & -36 & -15 & $29.45 \%$ \\
\hline $\begin{array}{l}\text { Compañia Minera Santa Luisa } \\
\text { S.A. }\end{array}$ & 11,927 & 13,238 & 74,184 & $8.12 \%$ & 7,214 & 4,713 & $39.51 \%$ \\
\hline $\begin{array}{l}\text { Compañia Minera San Ignacio } \\
\text { de Morococha S.A. }\end{array}$ & 3,595 & 4,546 & 79,704 & $5.63 \%$ & 58 & 3,537 & $98.39 \%$ \\
\hline Perubar S.A. & 4,341 & 4,301 & 65,054 & $8.12 \%$ & -981 & 5,322 & $122.61 \%$ \\
\hline Fosfatos del Pacifico S.A. & $-3,761$ & $-3,506$ & 66,833 & $8.12 \%$ & $-8,933$ & 5,172 & $-137.51 \%$ \\
\hline $\begin{array}{l}\text { Compañia Minera Atacocha } \\
\text { S.A. }\end{array}$ & $-8,721$ & $-6,886$ & 120,256 & $6.60 \%$ & $-14,825$ & 6,104 & $-69.99 \%$ \\
\hline $\begin{array}{l}\text { Sociedad Minera El Brocal } \\
\text { S.A.A. }\end{array}$ & 24,474 & 23,898 & 443,582 & $7.07 \%$ & $-7,457$ & 31,931 & $130.47 \%$ \\
\hline Minsur S.A. & 261,378 & 263,756 & $2,197,396$ & $7.54 \%$ & 98,179 & 163,199 & $62.44 \%$ \\
\hline $\begin{array}{l}\text { Compañia de Minas Buena } \\
\text { Ventura }\end{array}$ & 271,082 & 275,134 & 2,705,309 & $8.12 \%$ & 55,463 & 215,619 & $79.54 \%$ \\
\hline Total sector & $2,916,911$ & 2,990,307 & $15,811,488$ & $7.26 \%$ & $1,798,866$ & $1,118,045$ & $38.33 \%$ \\
\hline
\end{tabular}

De acuerdo con los resultados obtenidos según la Tabla 3 , los corolarios acumulados entre las 16 empresas pertenecientes al sector minero durante el periodo 2013, muestran que, en comparación con la utilidad neta, el EVA tuvo una diferencia absoluta de $\$ 1,307^{\prime} 008$ miles de dólares por debajo de la utilidad neta y una diferencia relativa del $61.10 \%$ menor que la utilidad neta; lo que indica que el sector minero en sus resultados acumulados a pesar de tener beneficio económico gracias a la utilidad neta no generó valor económico agregado, al mismo nivel que el beneficio económico obtenido; por tal motivo, el sector minero en el periodo 2013 generó un valor económico agregado equivalente al $38.90 \%$ de su beneficio económico acumulado (utilidad neta acumulada).

Tabla 3

Resultados con variación relativa y absoluta 2013

\begin{tabular}{|c|c|c|c|c|c|c|c|}
\hline Empresas & Utilidad & BAIDI & VCA & WACC & EVA & $\begin{array}{c}\text { Dif. } \\
\text { Absoluta }\end{array}$ & $\begin{array}{c}\text { Dif. } \\
\text { Relativa }\end{array}$ \\
\hline $\begin{array}{l}\text { Southern Peru Cooper } \\
\text { Corporation }\end{array}$ & 716,318 & 715,397 & $3,452,092$ & $8.69 \%$ & 415,410 & 300,908 & $42.01 \%$ \\
\hline $\begin{array}{l}\text { Shoughan Hierro Peru } \\
\text { S.A. }\end{array}$ & 295,244 & 309,834 & 727,328 & $6.82 \%$ & 260,235 & 35,009 & $11.86 \%$ \\
\hline $\begin{array}{l}\text { Volcan Compañía } \\
\text { Minera S.A.A. }\end{array}$ & 168,261 & 227,367 & $2,213,801$ & $7.05 \%$ & 71,225 & 97,036 & $57.67 \%$ \\
\hline $\begin{array}{l}\text { Sociedad Minera Cerro } \\
\text { Verde S.A. }\end{array}$ & 613,262 & 616,963 & $4,087,484$ & $8.69 \%$ & 261,761 & 351,501 & $57.32 \%$ \\
\hline $\begin{array}{l}\text { Compañia Minera } \\
\text { Milpo S.A. }\end{array}$ & 65,481 & 81,238 & 919,207 & $6.50 \%$ & 21,472 & 44,009 & $67.21 \%$ \\
\hline $\begin{array}{l}\text { Compañia Minera } \\
\text { Poderosa S.A. }\end{array}$ & 22,398 & 29,073 & 172,934 & $7.43 \%$ & 16,232 & 6,166 & $27.53 \%$ \\
\hline
\end{tabular}




\begin{tabular}{|c|c|c|c|c|c|c|c|}
\hline $\begin{array}{l}\text { Sociedad Minera Corona } \\
\text { S.A. }\end{array}$ & 16,715 & 21,436 & 69,752 & $7.63 \%$ & 16,112 & 603 & $3.61 \%$ \\
\hline $\begin{array}{l}\text { Minera Andina de } \\
\text { Exploraciones S.A.A. }\end{array}$ & 391 & 391 & 1,500 & $8.69 \%$ & 261 & 130 & $33.34 \%$ \\
\hline $\begin{array}{l}\text { Compañia Minera Santa } \\
\text { Luisa S.A. }\end{array}$ & $-1,424$ & $-2,503$ & 54,269 & $8.69 \%$ & $-7,219$ & 5,795 & $-406.95 \%$ \\
\hline $\begin{array}{l}\text { Compañia Minera San } \\
\text { Ignacio de Morococha } \\
\text { S.A. }\end{array}$ & $-12,107$ & $-7,972$ & 66,696 & $7.09 \%$ & $-12,700$ & 593 & $-4.90 \%$ \\
\hline Perubar S.A. & 6,974 & 7,322 & 90,515 & $7.40 \%$ & 623 & 6,351 & $91.07 \%$ \\
\hline Fosfatos del Pacifico S.A. & $-2,683$ & $-2,685$ & 81,628 & $8.69 \%$ & $-9,778$ & 7,095 & $-264.46 \%$ \\
\hline $\begin{array}{l}\text { Compañia Minera } \\
\text { Atacocha S.A. }\end{array}$ & $-14,024$ & $-13,111$ & 93,416 & $8.19 \%$ & $-20,760$ & 6,736 & $-48.03 \%$ \\
\hline $\begin{array}{l}\text { Sociedad Minera El Brocal } \\
\text { S.A.A. }\end{array}$ & $-7,709$ & $-4,835$ & 559,029 & $7.48 \%$ & $-46,674$ & 38,965 & $-505.45 \%$ \\
\hline Minsur S.A. & 234,955 & 244,284 & $2,382,780$ & $8.11 \%$ & 50,928 & 184,027 & $78.32 \%$ \\
\hline $\begin{array}{l}\text { Compañia de Minas } \\
\text { Buena Ventura }\end{array}$ & 37,004 & 42,050 & $2,613,666$ & $8.69 \%$ & $-185,078$ & 222,082 & $600.16 \%$ \\
\hline Total Sector & $2,139,056$ & $2,264,249$ & $17,586,097$ & $7.87 \%$ & 832,048 & $1,307,008$ & $61.10 \%$ \\
\hline
\end{tabular}

De acuerdo con los datos obtenidos según la Tabla 4, los resultados acumulados entre las 16 empresas pertenecientes al sector minero durante el periodo 2014, la utilidad neta en comparación al EVA tuvo una diferencia absoluta de $\$ 1,540$ '060 mil- les de dólares por debajo de la utilidad neta y una diferencia relativa del $117.17 \%$ menor que la utilidad neta; indicando que a pesar de tener beneficio económico a través de la utilidad neta no generó valor económico agregado durante el 2014.

Tabla 4

Resultados con variación relativa y absoluta 2014

\begin{tabular}{lrrrrrrr}
\hline Empresas & Utilidad & BAIDI & VCA & WACC & EVA & $\begin{array}{r}\text { Dif. } \\
\text { Absoluta }\end{array}$ & $\begin{array}{r}\text { Dif. } \\
\text { Relativa }\end{array}$ \\
\hline Southern Peru Cooper Corporation & 565,732 & 563,436 & $3,866,116$ & $9.75 \%$ & 186,490 & 379,242 & $67.04 \%$ \\
Shoughan Hierro Peru S.A. & 150,937 & 173,622 & 570,773 & $6.88 \%$ & 134,337 & 16,600 & $11.00 \%$ \\
Volcan Compañía Minera S.A.A. & 73,299 & 148,854 & $2,331,733$ & $7.54 \%$ & $-26,872$ & 100,171 & $136.66 \%$ \\
Sociedad Minera Cerro Verde S.A. & 377,606 & 375,691 & $4,917,939$ & $9.00 \%$ & $-67,004$ & 444,610 & $117.74 \%$ \\
Compañia Minera Milpo S.A. & 108,548 & 126,276 & $1,007,021$ & $7.43 \%$ & 51,447 & 57,101 & $52.60 \%$ \\
Compañia Minera Poderosa S.A. & 14,551 & 19,284 & 165,752 & $8.17 \%$ & 5,743 & 8,808 & $60.53 \%$ \\
Sociedad Minera Corona S.A. & 29,782 & 32,531 & 75,075 & $7.35 \%$ & 27,012 & 2,770 & $9.30 \%$ \\
Minera Andina de Exploraciones S.A.A. & -85 & -53 & 723 & $9.75 \%$ & -123 & 38 & $-45.29 \%$ \\
Compañia Minera Santa Luisa S.A. & 876 & 2 & 52,473 & $9.75 \%$ & $-5,114$ & 5,990 & $683.80 \%$ \\
Compañia Minera San Ignacio de & $-16,772$ & $-12,390$ & 52,142 & $8.73 \%$ & $-16,944$ & 172 & $-1.03 \%$ \\
Morococha S.A. & -584 & 464 & 101,074 & $7.47 \%$ & $-7,085$ & 6,501 & $1113.17 \%$ \\
Perubar S.A. & $-3,605$ & $-3,533$ & 74,190 & $9.75 \%$ & $-10,767$ & 7,162 & $-198.66 \%$ \\
Fosfatos Del Pacifico S.A. & $-2,240$ & $-1,268$ & 81,396 & $9.70 \%$ & $-9,165$ & 6,925 & $-309.13 \%$ \\
Compañia Minera Atacocha S.A. & 7,514 & 8,557 & 624,385 & $8.23 \%$ & $-42,812$ & 50,326 & $669.76 \%$ \\
Sociedad Minera El Brocal S.A.A. & 84,898 & 121,710 & $2,117,461$ & $8.63 \%$ & $-61,101$ & 145,999 & $171.97 \%$ \\
Minsur S.A. & $-76,065$ & $-45,540$ & $3,504,105$ & $9.65 \%$ & $-383,710$ & 307,645 & $-404.45 \%$ \\
Compañia de Minas Buena Ventura & $1,314,39$ & $1,507,643$ & $19,542,358$ & $8.61 \%$ & $-225,668$ & $1,540,060$ & $117.17 \%$ \\
\hline Total Sector & 2 & & & & & &
\end{tabular}


De acuerdo con los resultados obtenidos en la Tabla 5 , se observa que los resultados acumulados entre las 16 empresas pertenecientes al sector minero durante el periodo 2015, se evidencia que, en comparación con la utilidad neta, el valor económico agregado, tuvo una diferencia absoluta de $\$ 1,126$ ' 640 miles de dola- res por debajo de la utilidad neta y una diferencia relativa del $2718.33 \%$ menor que la utilidad neta; lo que implica que a pesar de que en sus resultados acumulados se ha tenido beneficio económico a través de la utilidad neta, no generó valor económico agregado.

Tabla 5

Resultados con variación relativa y absoluta 2015

\begin{tabular}{|c|c|c|c|c|c|c|c|}
\hline Empresas & Utilidad & BAIDI & VCA & WACC & EVA & $\begin{array}{c}\text { Dif. } \\
\text { Absoluta }\end{array}$ & $\begin{array}{c}\text { Dif. } \\
\text { Relativa }\end{array}$ \\
\hline $\begin{array}{l}\text { Southern Peru Cooper } \\
\text { Corporation }\end{array}$ & 336,756 & 330,460 & $4,116,409$ & $8.41 \%$ & $-15,730$ & 352,486 & $104.67 \%$ \\
\hline $\begin{array}{l}\text { Shoughan Hierro Peru } \\
\text { S.A. }\end{array}$ & 39,232 & 74,995 & 447,181 & $5.40 \%$ & 50,838 & $-11,606$ & $-29.58 \%$ \\
\hline $\begin{array}{l}\text { Volcan Compañía Minera } \\
\text { S.A.A. }\end{array}$ & 387,159 & 501,081 & $1,904,261$ & $6.32 \%$ & 380,825 & 6,334 & $1.64 \%$ \\
\hline $\begin{array}{l}\text { Sociedad Minera Cerro } \\
\text { Verde S.A. }\end{array}$ & 33,284 & 125,064 & $6,923,538$ & $6.28 \%$ & $-309,895$ & 343,179 & $1031.06 \%$ \\
\hline $\begin{array}{l}\text { Compañia Minera Milpo } \\
\text { S.A. }\end{array}$ & 67,001 & 84,672 & $1,034,592$ & $6.66 \%$ & 15,818 & 51,183 & $76.39 \%$ \\
\hline $\begin{array}{l}\text { Compañia Minera } \\
\text { Poderosa S.A. }\end{array}$ & 27,325 & 33,012 & 151,995 & $7.03 \%$ & 22,323 & 5,002 & $18.31 \%$ \\
\hline $\begin{array}{l}\text { Sociedad Minera Corona } \\
\text { S.A. }\end{array}$ & 7,532 & 10,125 & 74,093 & $7.12 \%$ & 4,851 & 2,681 & $35.59 \%$ \\
\hline $\begin{array}{l}\text { Minera Andina De } \\
\text { Exploraciones S.A.A. }\end{array}$ & 764 & 764 & 1,256 & $8.41 \%$ & 658 & 106 & $13.83 \%$ \\
\hline $\begin{array}{l}\text { Compañia Minera Santa } \\
\text { Luisa S.A. }\end{array}$ & $-3,490$ & $-5,215$ & 42,180 & $8.41 \%$ & $-8,762$ & 5,272 & $-151.07 \%$ \\
\hline $\begin{array}{l}\text { Compañia Minera San } \\
\text { Ignacio de Morococha } \\
\text { S.A. }\end{array}$ & $-10,368$ & $-4,159$ & 39,173 & $6.93 \%$ & $-6,874$ & $-3,494$ & $33.70 \%$ \\
\hline Perubar S.A. & $-2,582$ & $-2,100$ & 96,974 & $6.58 \%$ & $-8,477$ & 5,895 & $-228.32 \%$ \\
\hline Fosfatos Del Pacifico S.A. & $-2,997$ & $-2,648$ & 89,683 & $8.41 \%$ & $-10,190$ & 7,193 & $-240.02 \%$ \\
\hline $\begin{array}{l}\text { Compañia Minera } \\
\text { Atacocha S.A. }\end{array}$ & $-22,941$ & $-22,578$ & 58,266 & $8.41 \%$ & $-27,478$ & 4,537 & $-19.78 \%$ \\
\hline $\begin{array}{l}\text { Sociedad Minera El Brocal } \\
\text { S.A.A. }\end{array}$ & $-76,189$ & $-62,265$ & 575,483 & $6.87 \%$ & $-101,801$ & 25,612 & $-33.62 \%$ \\
\hline Minsur S.A. & $-421,830$ & $-388,817$ & $1,491,427$ & $7.22 \%$ & $-496,512$ & 74,682 & $-17.70 \%$ \\
\hline $\begin{array}{l}\text { Compañia de Minas } \\
\text { Buena Ventura }\end{array}$ & $-317,210$ & $-303,600$ & $3,393,396$ & $7.99 \%$ & $-574,788$ & 257,578 & $-81.20 \%$ \\
\hline Total Sector & 41,446 & 368,791 & $20,439,907$ & $7.28 \%$ & -1085194 & $1,126,640$ & $2718.33 \%$ \\
\hline
\end{tabular}

De acuerdo con los datos de la Tabla 6, los resultados acumulados de las $16 \mathrm{em}$ presas pertenecientes al sector minero durante el periodo 2016, se evidencia que, en comparación con la utilidad neta, el EVA tuvo una diferencia absoluta de \$1,158’506 miles de dólares por debajo de la utilidad neta y una diferencia relativa del $166.64 \%$ menor que la utilidad neta; indicando que a pesar de tener beneficio económico a través de la utilidad neta no generó valor económico agregado en la misma proporción. 
Tabla 6

Resultados con variación relativa y absoluta 2016

\begin{tabular}{|c|c|c|c|c|c|c|c|}
\hline Empresas & Utilidad & BAIDI & VCA & WACC & EVA & $\begin{array}{c}\text { Dif. } \\
\text { Absoluta }\end{array}$ & $\begin{array}{c}\text { Dif. } \\
\text { Relativa }\end{array}$ \\
\hline $\begin{array}{l}\text { Southern Peru Cooper } \\
\text { Corporation }\end{array}$ & 272,180 & 283,176 & $4,358,751$ & $8.19 \%$ & $-73,806$ & 345,986 & $127.12 \%$ \\
\hline $\begin{array}{l}\text { Shoughan Hierro Peru } \\
\text { S.A. }\end{array}$ & 62,063 & 68,058 & 335,310 & $6.51 \%$ & 46,218 & 15,845 & $25.53 \%$ \\
\hline $\begin{array}{l}\text { Volcan Compañía Minera } \\
\text { S.A.A. }\end{array}$ & 84,406 & 209,080 & $1,935,057$ & $6.58 \%$ & 81,773 & 2,633 & $3.12 \%$ \\
\hline $\begin{array}{l}\text { Sociedad Minera Cerro } \\
\text { Verde S.A. }\end{array}$ & 340,907 & 413,488 & $6,835,285$ & $6.53 \%$ & $-32,804$ & 373,711 & $109.62 \%$ \\
\hline $\begin{array}{l}\text { Compañia Minera Milpo } \\
\text { S.A. }\end{array}$ & 113,146 & 132,727 & $1,081,169$ & $6.61 \%$ & 61,292 & 51,854 & $45.83 \%$ \\
\hline $\begin{array}{l}\text { Compañia Minera } \\
\text { Poderosa S.A. }\end{array}$ & 52,618 & 55,991 & 190,090 & $7.28 \%$ & 42,150 & 10,468 & $19.90 \%$ \\
\hline $\begin{array}{l}\text { Sociedad Minera Corona } \\
\text { S.A. }\end{array}$ & 17,234 & 18,554 & 93,714 & $6.49 \%$ & 12,474 & 4,760 & $27.62 \%$ \\
\hline $\begin{array}{l}\text { Minera Andina De } \\
\text { Exploraciones S.A.A. }\end{array}$ & 715 & 738 & 948 & $8.19 \%$ & 660 & 55 & $7.64 \%$ \\
\hline $\begin{array}{l}\text { Compañia Minera Santa } \\
\text { Luisa S.A. }\end{array}$ & 7,257 & 7,602 & 50,144 & $8.19 \%$ & 3,495 & 3,762 & $51.84 \%$ \\
\hline $\begin{array}{l}\text { Compañia Minera San } \\
\text { Ignacio de Morococha } \\
\text { S.A. }\end{array}$ & 15,500 & 20,561 & 52,884 & $8.13 \%$ & 16,263 & -763 & $-4.92 \%$ \\
\hline Perubar S.A. & -224 & 965 & 95,169 & $6.82 \%$ & $-5,526$ & 5,302 & $-2366.85 \%$ \\
\hline Fosfatos del Pacifico S.A. & $-2,688$ & $-2,448$ & 100,161 & $8.19 \%$ & $-10,651$ & 7,963 & $-296.25 \%$ \\
\hline $\begin{array}{l}\text { Compañia Minera } \\
\text { Atacocha S.A. }\end{array}$ & 544 & 1,947 & 58,810 & $8.19 \%$ & $-2,870$ & 3,414 & $627.49 \%$ \\
\hline $\begin{array}{l}\text { Sociedad Minera El Brocal } \\
\text { S.A.A. }\end{array}$ & $-32,782$ & $-22,099$ & 590,153 & $7.15 \%$ & $-64,314$ & 31,532 & $-96.19 \%$ \\
\hline Minsur S.A. & 87,849 & 118,547 & $1,635,853$ & $7.16 \%$ & 1,361 & 86,488 & $98.45 \%$ \\
\hline $\begin{array}{l}\text { Compañia de Minas } \\
\text { Buena Ventura }\end{array}$ & $-323,492$ & $-296,505$ & $3,092,972$ & $7.84 \%$ & $-538,989$ & 215,497 & $-66.62 \%$ \\
\hline Total Sector & 695,233 & $1,010,382$ & $20,506,470$ & $7.38 \%$ & $-463,273$ & $1,158,506$ & $166.64 \%$ \\
\hline
\end{tabular}

Análisis inferencial

De acuerdo con los resultados obtenidos en la Tabla 7, existe relación alta positiva estadísticamente, significativa mediante la correlación del $r$ de Pearson $(r=.988$; con un $\mathrm{p}$ valor=.002); por tanto, si la utilidad neta de las empresas del sector minero crece, se verá reflejada en la generación de EVA, de manera similar si decrece la utilidad neta, en la misma medida lo hará el EVA.
Tabla 7

Análisis de relación entre utilidad y EVA, periodo 2012 al 2016

\begin{tabular}{llc}
\hline \multicolumn{2}{c}{ Correlaciones } & \\
& & EVA \\
\hline \multirow{3}{*}{ Utilidad } & Correlación de Pearson & $.988^{* *}$ \\
& $\mathrm{~N}$ & .002 \\
& $\mathrm{~N}$. (bilateral) & 5 \\
\hline
\end{tabular}

**. La correlación es significativa en el nivel 0,01 (2 colas). 
De acuerdo con los resultados obtenidos mediante el $r$ de Pearson existe correlación estadísticamente significativa positiva muy alta $(r=.977$; con un $p$ valor=.000), como se evidencia en la Tabla 8. Por consiguiente, existe un grado de relación positiva entre la utilidad neta y la generación de valor económico agregado, en las empresas del sector minero que cotizan en bolsa de valores del Perú durante el periodo 2012.

Tabla 8

Análisis de relación entre utilidad y VAN, periodo 2012

Correlaciones

\begin{tabular}{llc} 
& & VAN \\
\hline Utilidad & Correlación de Pearson & $.977^{* *}$ \\
& Sig. (bilateral) & .000 \\
& $N$ & 16 \\
\hline$* *$. La correlación es significativa en el nivel 0,01 (2 colas).
\end{tabular}

De acuerdo con los resultados obtenidos mediante el $r$ de Pearson, existe correlación significativa positiva muy alta $(r=$ .900 ; con un $p$ valor $=.000$ ), como se muestra en la Tabla 9. Por consiguiente, existe un grado de relación positivo entre la utilidad neta y la generación de valor económico agregado, en las empresas del sector minero que cotizan en bolsa de valores del Perú durante el periodo 2013.

Tabla 9

Análisis de relación entre utilidad y EVA, periodo 2013

\begin{tabular}{llc}
\hline \multicolumn{2}{c}{ Correlaciones } & EVA \\
\hline \multirow{3}{*}{ Utilidad } & Correlación de Pearson &, $900^{* *}$ \\
& Sig. (bilateral) & .000 \\
& $N$ & 16 \\
\hline **. La correlación es significativa en el nivel 0,01 (2 colas).
\end{tabular}

De acuerdo con los resultados obtenidos mediante el $r$ de Pearson, existe correlación estadísticamente significativa positiva media ( $r=.501$; con un $p$ valor=.024), como se muestra en la Tabla 10. De modo que, se determina la existencia de un grado de relación positivo entre la utilidad neta y la generación de valor económico agregado, en las empresas del sector minero que cotizan en bolsa de valores del Perú durante el periodo 2014.

Tabla 10

Análisis de relación entre utilidad y EVA, periodo 2014

Correlaciones

\begin{tabular}{llc} 
& & EVA \\
\hline Utilidad & Correlación de Pearson & $.501^{* *}$ \\
& $\mathrm{~N}$ & .024 \\
& 16 \\
\hline$* *$. La correlación es significativa en el nivel 0,01 (2 \\
colas).
\end{tabular}

De acuerdo con los resultados obtenidos mediante el $r$ de Pearson, existe correlación significativa positiva alta $(r=.829$; con un $p$ valor $=.000$ ), como se muestra en la Tabla 11. Confirmando que existe un grado de relación positivo entre la utilidad neta y la generación de valor económico agregado en las empresas del sector minero que cotizan en la bolsa de valores del Perú durante el periodo 2015.

Tabla 11

Análisis de relación entre utilidad y EVA, periodo $\underline{2015}$

\begin{tabular}{llc}
\hline & \multicolumn{2}{c}{ Correlaciones } \\
& & EVA \\
\hline \multirow{3}{*}{ Utilidad } & Sig. (bilateral) & $.829^{* *}$ \\
& $\mathrm{~N}$ & .000 \\
& & 16 \\
\hline
\end{tabular}

**. La correlación es significativa en el nivel 0,01 (2 colas). 
De acuerdo con los resultados obtenidos mediante el $r$ de Pearson, existe correlación significativa positiva alta $(r=.613$; con un $\mathrm{p}$ valor=.006), como se muestra en la Tabla 12. Por tanto, se determina que existe un grado de relación positivo entre la utilidad neta y la generación de valor económico agregado, en las empresas del sector minero que cotizan en la bolsa de valores del Perú durante el periodo 2016.

Tabla 12

Análisis de relación entre utilidad y EVA, periodo 2016

Correlaciones

\begin{tabular}{llc} 
& & EVA \\
\hline \multirow{3}{*}{ Utilidad } & Correlación de Pearson & $.613^{* *}$ \\
& Sig. (bilateral) & .006 \\
& $\mathrm{~N}$ & 16 \\
\hline
\end{tabular}

**. La correlación es significativa en el nivel 0,01 (2 colas).

\section{Discusión y Conclusión}

La utilidad neta acumulada y el valor económico agregado acumulado EVA, de las empresas del sector minero durante el período comprendido entre los años 2012 al 2016, presentan una relación positiva muy alta, lo que implica que a medida que las utilidades incrementan, el EVA también lo hace; no obstante, según el estado del análisis de resultados acumulados, se determinó que pese a tener un resultado positivo en utilidad neta, el reducto no garantiza la existencia de un incremento de valor económico agregado, en el mismo periodo; dados los diversos condicionantes que tiene la determinación del valor económico agregado, como son el beneficio antes de intereses y después de impuestos, el valor contable del activo y el costo promedio ponderado del capital. Puesto que cada uno de estos componentes a su vez tienen repercusión en otros factores adicionales. 


\section{Referencias}

Acuña, G. (2001). EVA Valor económico agregado. https://www.gestiopolis.com, https://www.gestiopolis.com/eva-valoreconomico-agregado/.

Amat, O. 2. (1999). EVA valor económico agregado 1999. Barcelona: Norma.

Amat, O. (2000). Madrid: Gestion.

Apaza, M. (2005). Contabilidad Estrategica del EVA. Lima: Real.

Backes, J. (2002). EVA Valor Económico Agregado.

BCRP. (2013). Memoria Anual. Lima

BCRP. (2014). Memoria Anual. Lima.

BCRP. (2015). Memoria Anual. Lima.

BCRP. (2016). Memoria Anual. Lima

Benavidez, R. (2012). La Mineria Responsable y sus Aportes al Desarrollo del Perú. Lima: Minera Buena Ventura.

Copeland, T. (1990). Measuring and managing the value of companies. Nueva York: Jonh Wiley\&Sons.

Corporation, S. P. (2012). Memoria anual. Lima.

Corporation, S. P. (2013). Memoria Anual. Lima.

Corporation, S. P. (2014). Memoria Anual. Lima.
Crespo, T. e. (2003). Administración de empresas. Madrir: MAD.

Dodd, J. (1996). EVA a new panacea? Businessand Economic Review, 26-28.

Estupiñan, G. \&. (2006). Analisis de Estados Financieros y de Gestion. Bogota Colombia: Bogota.

Hernandez, S., \& Baptista, L. (2010). Metodologia de la Investigacion. Mexico DF: McGRAW-HILL / INTERAMERICANA EDITORES, S.A. DE C.V.

Li Bonilla, F. (13 de 03 de 2018). Dinero. Obtenido de http://www.dinero.com/empresas/articul o/que-es-el-eva-y-como-secalcula/239711

MEF. (2013). Informe de Evaluacion Semestral del Plan Estartegico Institucional. Lima.

Morales, C. A. (2011). Estado de resultados. En C. A. Morales, Contabilidad Financiera (pág. 99). Veracruz: Universidad Veracruzana.

Navarro, J. D. (2017). ADMINISTRACIÓN FINANCIERA - Costo promedio de capital (WACC). https://www.abcfinanzas.com, https://www.abcfinanzas.com/administra cion-financiera/costo-promedio-decapital-wacc.

Ochoa, S. (2002). Administracion Financiera. Mexico: McGraw-Hill.

Ortiz, H. (2006). Analisis Financiero Aplicado. Bogota: Universidad Externado de Colombia. 
Stewar, G. (1999). "La busqueda del Valor: Guia de aplicacion del EVA". Gestion 2000.

Steward, S. (2017). The EVA Company. http://sternvaluemanagement.com, http://sternvaluemanagement.com/intell ectual-property-joel-stern/proprietarytools-value-creation/.
Urias, V. (1995). Analisis de Estados Financieros. España: Ed. McGraw Hill

Verde, C. (2016). Memoria Anual. Lima.

Volcan. (2015). Memoria Anual. Lima. 